severe and often persisted for $>35$ days. FCM, but not IIM, induced changes in vitD and calcium homeostasis that triggered secondary hyperparathyroidism, which likely contributed to persistence of hypophosphatemia. Alteration of bone metabolism after FCM administration may be of great clinical significance particularly for IBD patients who often have low vit D and regularly receive iv iron.

\section{PTH-138 COLORECTAL CANCER, COLECTOMY AND INFLAMMATORY BOWEL DISEASE ACTIVITY FOLLOWING LIVER TRANSPLANTATION IN PRIMARY SCLEROSING CHOLANGITIS}

${ }^{1}$ Tom Thomas*, ${ }^{2}$ Rachel Cooney, 2,3 Tariq Iqbal, 2,35ubrata Ghosh, 2,3 James Ferguson, ${ }^{4}$ ProfGideon Hirschfield, $2,3,5$ Palak Trivedi. ${ }^{1}$ University Of Oxford, Oxford, UK; ${ }^{2}$ University Hospitals Birmingham, Birmingham, UK; ${ }^{3}$ National Institute for Health Research (NIHR) Birmingham Biomedical Research Centre (BRC), Birmingham, UK; ${ }^{4}$ Toronto Centre for Liver Disease, University of Toronto, Toronto, Canada; ${ }^{5}$ University of Birmingham, Birmingham, UK

\subsection{6/gutjnl-2019-BSGAbstracts. 197}

Introduction Primary sclerosing cholangitis[PSC] is the classical hepatobiliary manifestation of inflammatory bowel disease[IBD] for which liver transplantation[LT] is the only curative therapy.In this study,we provide pooled incidence rates[IR] and time trends of (1)colorectal cancer[CRC], (2)flares in IBD activity and (3)colectomy rates post LT, through systematic review and meta-analysis.

Methods A systematic literature search of Medline and Embase was undertaken to identify pertinent studies from 1981 to 2014.Included studies were assigned to one or more of the three analytical streams. The 'meta' package (R Studio (V.1.1.463)) and Revman was used to pool IRs and HRs from individual studies using a random effects model respectively.

Results 42 studies qualified for inclusion in the systematic review.20 studies detailing the clinical course of 1994 patients (cumulative 9874 patient years(PY)) were pooled to assess the occurrence of dysplasia or CRC(combined endpoint) and CRC only; yielding an IR of 14.97 (95\%CI9.74-23.02) and 9.21 (95\%CI6.01-14.09) per 1000 PY,respectively. Heterogeneity was considerable $\left(\mathrm{I}^{2}=86 \%\right)$. The incidence of post LT CRC was seen to be decreasing over the past decade(figure 1A).The estimated colectomy rate following OLT was IR 23.18(95\% CI16.74-32.08, I ${ }^{2}=84 \%$ ) per 1000 PY. IR for colectomy due to dysplasia/CRC was 11.25 per 1000 PY(95\%CI6.43-19.68) and decreasing over time(figure 1B).By contrast, deterioration in IBD activity necessitating colectomy was noted for IR 13.26 per 1000 PY(95\% CI 9.95-17.66), with no change in the incidence rate over study duration(figure 1C).9 studies reported on post LT clinical course of IBD according to endoscopy findings or need for escalation in IBD therapy.27.6\% of patients $(n=584)$ experienced deterioration in IBD activity after LT.The effect of ursodeoxycholic acid [UDCA] on the risk of CRC after LT was examined by 3 studies.Due to data availability,only 2 studies could be pooled. Use of UDCA increased the risk of CRC post LT in the PSC cohort; HR 2.90 (95\%CI 1.37-6.11).Studies examining the impact of UDCA on colectomy and IBD activity post-LT were inconclusive.

Conclusion The heightened risk of CRC mandates ongoing colonoscopic surveillance in the PSC/IBD LT population, although the incidence appears to be decreasing.Identification of risk predictors impacting the course of IBD is of critical importance,given that deterioration in activity appears to be the principal indication for colectomy post LT.

\section{Liver \\ Orals}

\section{OTH-10 THERAPEUTIC INTERLEUKIN 4 MODULATES MONOCYTE DYNAMICS AND ACCELERATES REPAIR FOLLOWING ACUTE LIVER INJURY}

${ }^{1}$ Ruairi Lynch*, ${ }^{1}$ Calum Bain, ${ }^{1}$ Cathy Hawley, ${ }^{2}$ Professor Stuart Forbes, 'Steve Jenkins. 'Queen's Medical Research Institute, University of Edinburgh, Edinburgh, UK; ${ }^{2}$ Centre for Regenerative Medicine, University of Edinburgh, Edinburgh, UK

\subsection{6/gutjnl-2019-BSGAbstracts.198}

Introduction Acute liver failure has significant mortality, and in its most severe form, the only treatment is liver
A) Colorectal cancer and dysplasia

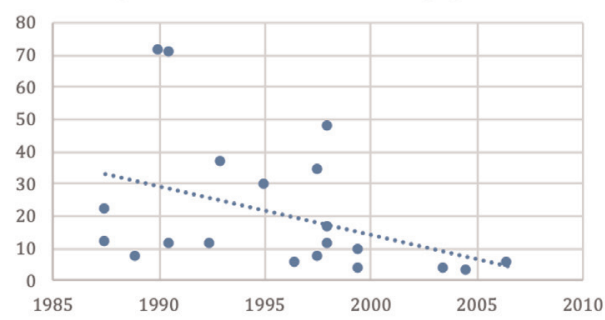

C) Colectomy due to IBD activity refractory to medical therapy

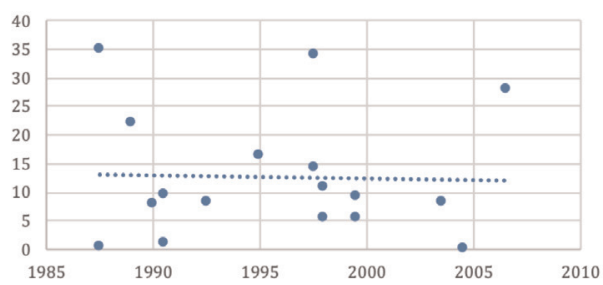

B) Colectomy due to CRC

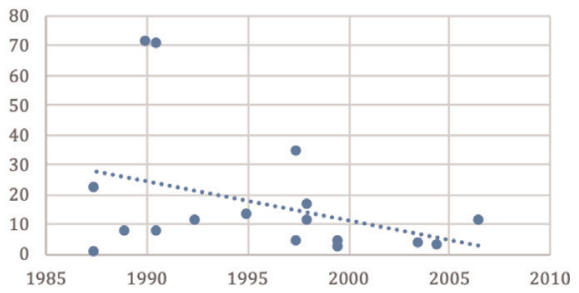

Abstract PTH138 Figure 1 Time trend analysis of incidence rates (per 1,000 pt.-yrs.) of IBD complications in PSC liver transplantation 